\title{
Wetting and spreading of human blood: recent advances and applications
}

\author{
F. R. Smith \\ Aix-Marseille University, IUSTI UMR CNRS 7343, Marseille, France \\ D. Brutin \\ Aix-Marseille University, IUSTI UMR CNRS 7343, Marseille, France \\ Institut Universitaire de France, IUF, Paris, France
}

\begin{abstract}
Investigation of the physical phenomena involved in blood interactions with real surfaces present new exciting challenges. The fluid mechanical properties of such a fluid is singular due its non-Newtonian and complex behaviour, depending on the surrounding ambient conditions and the donor/victim's blood biological properties. The fundamental research on the topic remains fairly recent; although it finds applications in fields such as forensic science, with bloodstain pattern analysis, or biomedical science with the prospect of disease detection from dried blood droplets. In this paper, we review the understanding that has been achieved by interpreting blood wetting, spreading and drying when in contact, ex-vivo, with non-coated surfaces. Ultimately, we highlight the applications with the most up to date research, future perspectives, and the need of advancing further in this topic for the benefit of researchers, engineers, bloodstain pattern analysts, and medical practitioners.
\end{abstract}

Keywords: Drop, evaporation, interfaces, complex fluid, rheology

\section{Introduction}

The interaction of blood with foreign, non-coated, surfaces is an emerging research subject that finds various applications in fields such as forensic science or biomedi5 cal discipline. Although blood flow circulation is a major subject that was thoroughly studied, interest in blood exvivo, i.e. outside the body, and its interaction with a noncoated surface, is less significant. However the properties of blood as a complex, biological, colloidal fluid, differ from 10 those of more commonly studied, pure or complex, fluids, stimulating a recent curiosity concerning the behaviour of blood with real porous and non-porous surfaces. While applied studies on the topic had already been made, in 2011 the work on the evaporation of blood droplets by

15 Brutin et al. gave a fundamental insight into blood drying pattern dynamics 4]. This work was later completed by recent studies whose major findings are presented in table 1. When blood is in contact with a substrate the two main interface transfers are first the wetting, and then the evaporation. While wetting accounts for the equilibrium of the various applied forces at the triple line (meeting point of blood, surface, and air), evaporation describes the phase change taking place after as recalled by the review

\footnotetext{
* Corresponding author

** Corresponding author

Email addresses: fiona.smith@univ-amu.fr (F. R. Smith), david.brutin@univ-amu.fr (D. Brutin)
}

of Sefiane et al. of 2011 26. These processes are dependent on variables such as ambient temperature, humidity, and pressure, wettability and roughness of the surface, time elapsed since blood deposition, volume and blood's clotting response. Indeed, when blood is outside the body, its properties change rapidly due to platelet activation leading to the coagulation cascade response. The importance of understanding this problem for forensic science and biomedical applications, appears to be crucial since it is used as a tool to obtain evidence in crime scene reconstitution or in medical diagnosis. The National Institute of Standards and Technology (NIST) has pointed out, in a very recent report, the urge of using valid scientific methods before presenting evidence in courtrooms 22. This illustrates the demand that exists concerning this research. To respond, we investigated the current understanding of blood physical and mechanical properties, and the most up to to date advances in the field. In this paper, we present first the characteristics of blood, then its spreading behaviour over dry porous and non-porous surfaces, followed by its drying dynamics, and finally we review the physics of impacting droplets. At last, we discuss the implications that the understanding of blood behaviour on real surfaces has for applications, and the future challenges that research is confronted with. 


\begin{tabular}{|l|l|l|l|l|}
\hline First Author & Year & Discipline & $\begin{array}{l}\text { Fundamental / } \\
\text { applied science }\end{array}$ & Major findings \\
\hline \hline Hudevoca & 2017 & Haematology & Applied science & $\begin{array}{l}\text { Feasibility of prenatal detection of haemophilia based } \\
\text { on maternal blood droplet [12. }\end{array}$ \\
\hline Comiskey & 2017 & Forensic & Applied science & $\begin{array}{l}\text { Theoretical model for predicting bloodstain patterns } \\
\text { from back spatter from blunt bullet gunshot [7]. }\end{array}$ \\
\hline Totesbury & 2017 & Forensic & Applied science & $\begin{array}{l}\text { Dynamic of blood wetting on hard surfaces using a syn- } \\
\text { thetic blood substitute[30]. }\end{array}$ \\
\hline Laux & 2016 & Acoustics & $\begin{array}{l}\text { Fundamental } \\
\text { science }\end{array}$ & $\begin{array}{l}\text { Ultrasounds can be used in order to monitor the rheo- } \\
\text { logical properties of blood[20. }\end{array}$ \\
\hline Cho & 2015 & Forensic & Applied science & $\begin{array}{l}\text { Differentiation between transfer patterns and spatter } \\
\text { patters onto fabrics[6]. }\end{array}$ \\
\hline Laan & 2015 & Forensic & $\begin{array}{l}\text { Fundamental } \\
\text { science }\end{array}$ & $\begin{array}{l}\text { Fluid dynamic model to estimate the point of origin of } \\
\text { blood spatters, taking into account gravity and drag[18]. }\end{array}$ \\
\hline
\end{tabular}

Table 1: References of special interest studies published over the last three years

\section{Blood properties}

\subsection{Biological composition}

Blood is categorised as a body fluid that accounts for roughly $7 \%$ of the human body weight. It has two main constituents: plasma, the fluid medium and blood cells, 90 the colloids. Plasma is a water based solution, composed 55 for $92 \%$ of water, which dissolves and transports organic and inorganic molecules, and for $8 \%$ of dissolved solutes [3. The solutes are mainly sodium electrolytes; nutrients and organic wastes are found as well in diverse amounts, 95 and finally the proteins, which account for 7-9\% of the

60 plasma. These proteins are albumins (80\%), globulin (16\%) and fibrinogen (4\%) that is really important in blot clot formation. Fibrinogen is a globulin of very high molecular weight synthesised exclusively from the liver that can be en $_{100}$ precipitated easily. During coagulation, the fibrin coming from fibrinogen forms a web leading to the clot formation. The blood cells are divided into three categories, the red blood cells (RBCs) that transport oxygen and carbon dioxide, the white blood cells (WBCs) (neutrophils and monocytes, eosinophils, basophils and lymphocytes), and

70 the platelets, which play an important role in the clotting ${ }_{105}$ response. Indeed platelets prevent bleeding by clumping and clotting vessel injuries. Thus all these constituents are main parameters to understand this complex, nonNewtonian, fluid, since an excess or a lack of these com75 ponents would alter its wetting and its spreading.

\subsection{Physical and rheological properties}

Blood has a small density variation between 1020 and $1060 \mathrm{~kg} \cdot \mathrm{m}^{-3}$, due to the differences in the individuals haematocrit level, which is defined as the ratio of $\mathrm{RBCs}_{115}$ volume to whole blood volume. The surface tension of blood is known to be similar to that of water, as shown by the work of Brutin et al. where they found $\theta=69.8$ $\mathrm{mN} . \mathrm{m}^{-1}$ as average statistical surface tension. Studies, such as the one of Chao et al. of 2014, account for the ${ }_{120}$
85 shear thinning properties of blood, since its viscosity decreases to a constant value at high shear rates $(\eta=4.8$ mPa.s), although it spreads like Newtonian fluids of similar viscosity. Rheological properties of a liquid are determined by the liquid viscosity, $\mu$, and the applied shear rate, $\dot{\gamma}$, according to the following relationship: $\mu=k \dot{\gamma}^{n-1}$, where $\mathrm{n}<1$ corresponds to pseudoplastic fluids. The is in an agreement with previous measurements of blood rheology [5]. As described by Baskurt et al., the apparent viscosity depends on the existing shear forces, and is determined by its biological properties: haematocrit, plasma viscosity, RBCs aggregation, and the mechanical properties of RBCs[1]. Alteration of those properties, such as a noteworthy modification of the haematocrit value, accounts for the heamorheological variations. Additionally, the viscoelastic properties of blood come from the high deformability of the RBCs.

\section{Spreading of complex fluids}

For a lot of applications (like painting, coating...), a smooth deposition is critical. However, only a few studies were published on the understanding of the influence of complex fluids on the dynamics of spreading. The most obvious way to improve spreading for aqueous solutions is to add surfactants to decrease the liquid-vapour interfacial tension and increase the initial spreading coefficient. Rafaï super-spreading of aqueous surfactants drops on hydrophobic surfaces. The super-spreading is due to a large affinity of the surfactant molecules for the solid substrate. If the surfactant molecules are transported rapidly and efficiently over the (small) height of the droplet in order to saturate the solid surface, this entails a large surface tension gradient over a small distance, and consequently a large Marangoni force. This, in turn, leads to the linear time evolution of the radius. The difference with the 'classical' surfactants observed here is then either a difference in affinity for the 


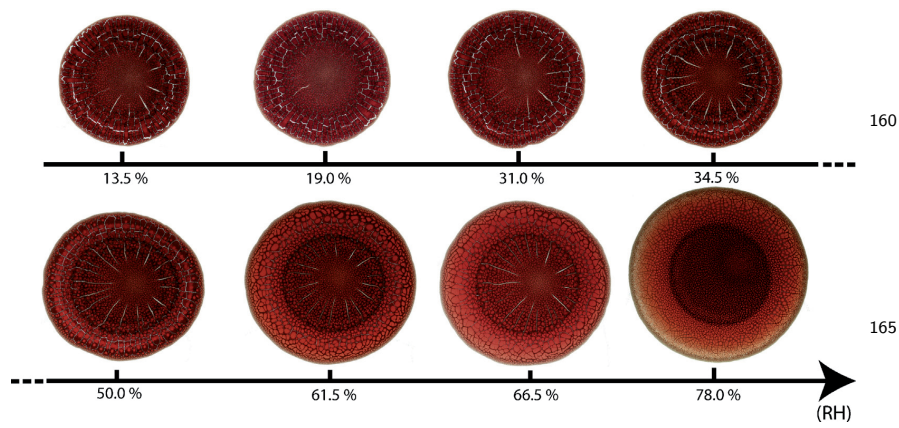

Figure 1: Top-view images of drop deposits left after complete evaporation of sessile drops of whole blood (same scale). All experiments are performed for a same drop volume $(\mathrm{V}=14.2 \mu \mathrm{L})$ and increasing ${ }^{170}$ RH (microscope ultraclean glass substrate, room temperature: 23.8 ${ }^{\circ} \mathrm{C}$, room pressure: $1005 \mathrm{hPa}$ ), reproduced from [2] with permission from Elsevier, copyright 2013.

solid substrate or a difference in the transport efficiency ${ }^{175}$ to that same substrate, a question that remains to be answered. In 2013, Bouzeid and Brutin [2] studied the influence of relative humidity $(\mathrm{RH})$ on the spreading behaviour and pattern formation of a human blood droplet. The drops of blood of same volume are deposited on $\mathrm{an}^{180}$ ultra-clean glass substrate and evaporated inside a humidity chamber with a controlled range of $\mathrm{RH}$ between $13.5 \%$ and $78.0 \%$. Their experiments show that the contact andecreases as a function of the $\mathrm{RH}$ which influences the final deposition pattern at the end of the evaporation pro- ${ }^{185}$ cess (Fig. 1). Due to the effect of RH on the contact angle of the drop of blood, initial evaporative rate is dependent of $\mathrm{RH}$ values. They observed cracks pattern at the end 135 of the drying process which is due to the competition between the drying regime and the gelation inside the drop ${ }^{190}$ of blood. Indeed, at first, the Marangoni convection inside the droplet induces the transport of particles towards the rim, and thus favours evaporation at the triple line. This

140 corresponds to a convective evaporation. Later, once the particles concentration reaches a critical point, gelation ${ }^{195}$ occurs, and evaporation occurs through the porous media. The transition between the purely convective evaporation regime and the gelation regime appears always at $65 \%$ of

145 the total drying time. Thus, controlling evaporative rate by evaporating drops of blood at different $\mathrm{RH}$ levels im- ${ }^{200}$ pacts strongly the wettability properties and the final pattern of drying drops of blood. The influence of $\mathrm{RH}$ on the final pattern of a dried drop of human blood is of huge im-

150 portance for biomedical applications where drops of blood are drying in an open atmosphere.

\section{Patterns in dried drops and pools}

\subsection{Patterns in dried drops}

Martusevich and Bochkareva presented in 2007 some $_{210}$

first results concerning the morphology of dried blood serum droplets with viral hepatitis 23 . Out of the 58 people included in the study, 32 were healthy, 14 patients had viral hepatitis B and 12 patients had viral hepatitis C. The authors established that the presence of viral hepatitis in patient's organism significantly changes the result of free and initiated blood serum crystallization. They concluded on the feasibility of single out the biological fluid crystallogenesis characteristics according to virus type. Before 2011, published studies were performed on blood serum. Brutin et al. achieved in 2011 the first observations of the Marangoni flow motion inside a drop of human whole blood using a digital camera [4]. They showed that the motion of RBCs at the edge of the drop is the main mechanism of blood drop deposition and early evaporation, since Marangoni convection favours RBCs displacement towards the rim and evaporation at the triple line. The mechanisms involved in the blood drop evaporation are confirmed through the evaporation mass flux, which is measured to be in agreement with pure water drop evaporation. Other biological elements (white blood cells, proteins) are transported to the edge of the drop with the RBCs and contribute to the pattern formation and the deposit wettability with the substrate and the mechanical properties. The blood evaporation dynamic is comparable to the evaporation of pure fluids of similar mass concentration in colloids. Totally different patterns are formed according to whether the person is healthy or suffers from anaemia or hyperlipidaemia. A few years later, in 2014, Sobac and Brutin evidenced the influence on the final pattern of the wetting of blood on a substrate 28 . They showed that all of the mechanisms are linked, and the change of wettability highly affects the deposit shape. The evaporation of a drop of blood with an initial angle of $90^{\circ}$ revealed the formation of a complex morphology due to the presence of an elastic buckling instability. In this situation, a shell forms during the drying due to the accumulation of particles at the free surface, and is deformed by buckling, leading to the formation of folds. The final shape is no longer axisymmetric. The different behaviours encountered were assimilated to a stability diagram obtained for physical suspensions, and a good level of agreement was observed. The results presented are notably close to those obtained after the evaporation of drops of bovine serum, suspensions of colloidal particles and suspensions of polymers. The practical application of pattern formation in drying drops of whole blood requires a complete understanding of the involved mechanisms such as spreading, wetting, evaporation heat and mass transfer, and cracks formation.

\subsection{Patterns in dried pools}

Although the drying of gels, or colloidal Newtonian suspensions has been largely studied, research on the drying of blood pools remains scarce. Unlike droplets, in the case of a pool, gravitational forces are more important than surface tension forces; this induces the flattening of the liquid on the top part. One of the first considerations to observe concerning drying blood is that the properties of the blood change when the blood is ex-vivo. Visually the change 
of colour from bright red to dark brown due to the oxiox-haemoglobin $\left(\mathrm{HbO}_{2}\right)$ to methaemoglobin (met-Hb) and haemachrome (HC), is a first indicator. Another important step when bleeding occurs, is haemostasis, which involves coagulation corresponding to the change of blood from a liquid into a gel [10]. In 2016, Laan et al. vestigated the topic, and observed the different phases that a drying pool of blood goes through and arbitrarily called them coagulation stage, gelation stage, rim desiccation stage, centre desiccation stage, and final desiccation stage [19]. Comparatively to a drying droplet, their experafter drying, which corresponds to the colloids, mainly RBCs, present in blood. When whole blood is deposited on a foreign surface, at first the RBCs are homogeneously distributed. Then rapidly coagulation takes places. Dur230 ing this phase the fibrin coming from the fibrinogen forms a web leading to the clot formation. The evaporation that follows leads to the displacement of the cells not trapped yet in the web towards the pool's rim. The drying front starts at the rim of the pool and goes towards the cen235 tre of the pool in a non-uniform way. The stress induced by the water evaporation leads to the noteworthy cracks observed on dried blood pools. In 2017, Ramsthaler et al. compared the drying processes of extra-corporal blood without any additives to blood that had received anticoagulation therapy for small volume droplets by studied the wipeability of bloodstains. As a result, they found that the time of the drying process was delayed more efficiently by anticoagulation therapy than it was by antithrombotic drugs or platelet aggregation inhibitors, meaning that the ast part of the coagulation process is of high importance on the drying dynamics compared to the primary stages of the haemostasis when platelet activation and aggregation take place 25 .

\section{Drop impact on solid surfaces}

\subsection{Impact theory}

When a drop of blood is released onto a dry solid surface from different heights, it impacts with energy due to the velocity of the fall. Understanding the correlation between a blood drop pattern and its velocity of fall is im- ${ }_{285}$ 25 portant to the forensic community as it could answer some crime scene reconstruction questions. In 2014, Laan et al. 17 described the phenomenon, and how inertia forces favour spreading, but adhesion and viscous forces counter this spreading so that the drop reaches a maximum spreaddiameter commonly named $D_{\max }$, as shown on figure 2. It normally remains self-pinned to the surfaces before evaporation and drying mechanisms take place. The following resulting outcome of the drop stain depends upon the drop, the surface and the surrounding properties. To define the impact dynamics, the dimensionless Weber and Reynolds numbers are commonly used since they describe the liquid properties and the impact. The Weber number

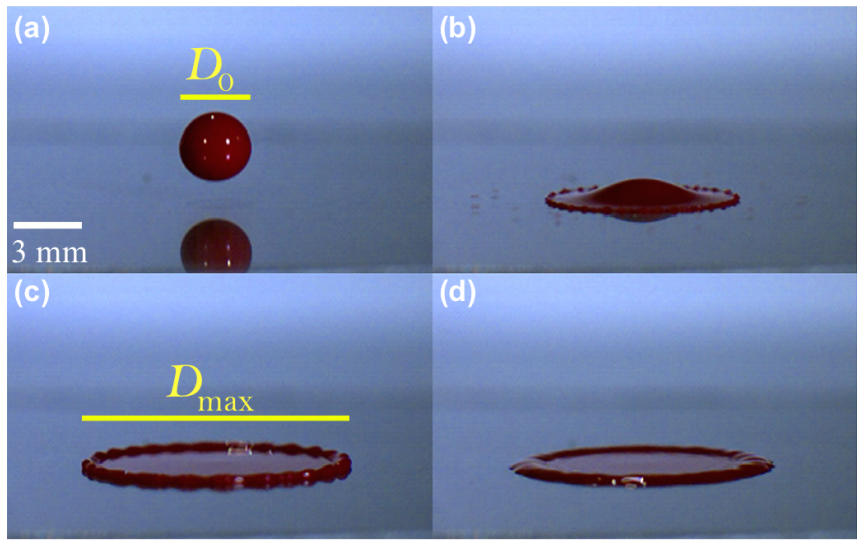

Figure 2: (a)-(d) High-speed camera recordings of a single blood droplet impacting at $2 \mathrm{~m} . \mathrm{s}^{-1}$ on a stainless-steel substrate. (a) At $0.2 \mathrm{~ms}$ prior to droplet impact, the droplet is spherical. (b) At $0.6 \mathrm{~ms}$ after droplet impact on the surface, a thin lamella spreads outwards due to the inertial forces. (c) At $2.4 \mathrm{~ms}$ after droplet impact, the lamella increases in size. Spreading of the lamella slows down until it reaches its maximum size $\left(D_{\max }\right)$, while there is a buildup of liquid in the outer rim. (d) At $4 \mathrm{~ms}$ after droplet impact, the bulk of the liquid is distributed over the entire stain. Reproduced from [17] with permission from APS, copyright 2014

( We $\left.=\rho D_{0} U^{2} / \sigma\right)$, is the ratio between capillary and inertial forces whereas the Reynolds number $\left(\operatorname{Re}=\rho D_{0} U / \eta\right)$ is the ratio between the viscous and the inertial forces. $\rho$, $\eta$ are respectively the blood density and viscosity. $\sigma$ is the blood/air surface tension. U stands for the impact velocity of the droplet, and $D_{0}$ for the initial drop diameter. One of the latest model describing the maximum spreading diameter of blood droplet, is the model of 2016 of Lee et al. 21, who presented a universal solution taking into account that energy conservation is the only physical principal needed to describe the impact behaviour of droplets, according to the following equation:

$$
\left(\beta_{\max }^{2}-\beta_{0}^{2}\right)^{1 / 2} R e^{-1 / 5}=\frac{W e^{1 / 2}}{A+W e^{1 / 2}},
$$

with $\beta_{\max }$ the maximum spreading ratio of the droplet (the ratio of the maximum spreading diameter by the initial diameter of the given droplet), $\beta_{0}$ the maximum spreading ratio at zero impact velocity for the same surface (the ratio of the maximum spreading diameter by the initial diameter of the droplet at $0 \mathrm{~m} . \mathrm{s}^{-1}$ ), and $\mathrm{A}=7.6$, the fitting constant.

\subsection{Surface influence}

Interest for various industrial applications, led many researches to study the influence of the surfaces properties on the impact outcomes of impacting droplets of various Newtonian fluids, as thoroughly described in the recent review of Josserand and Thoroddsen [15. Meanwhile, in 2016, Kim al. investigated the influence of the target properties on the trajectory reconstruction of blood droplets impacting different surfaces using glass, polycarbonate bare, aluminium mirror and cardstock [16]. To 

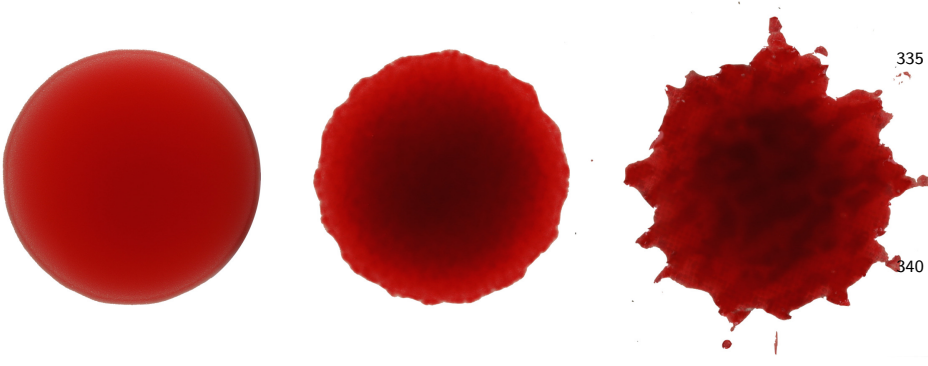

Increasing average surface roughness

for impact velocity of $2.6 \mathrm{~m} . \mathrm{s}^{-1}$

Figure 3: Deformation pattern of three blood drip stains of same initial volume $\left(V=13.8 * 10^{-3} \mathrm{~mL}\right)$ impacting at $U=2.6 \mathrm{~m} . \mathrm{s}^{-1}$ surfaces of increasing roughness. Reproduced from [27] with permission from APS, copyright 2017.

quantify the influence, they correlated the Ohnesorge number $(\mathrm{Oh}=\sqrt{W e} / \mathrm{Re})$, which relates the energy of the impact to the resistant work of surface tension and viscous forces, to the spreading ratio. They found that an increase 355 in surface roughness reduces the spreading upon drop impact, which led them to conclude that the stain size cannot directly be related to an impact velocity, which supports the model of Lee et al. that takes into account the initial spreading ratio of the liquid at a zero impact velocity for ${ }^{360}$ a given surface, implying that this ratio is substrate dependent. This suggests that not only the spreading could be influenced by the target properties, but the outcome, and whether splashing can be observed or not. Recently Smith et al. investigated the influence of the surface prop-365 erties on the splashing threshold of blood drip stains 27. In their study they defined the splashing threshold as the minimum drop velocity inducing the drop to break-up and have at least one secondary droplet. The experimental results showed that an increase in surface roughness ${ }^{370}$ leads to an earlier splashing threshold when comparing the Weber number of their impacting drops with the average surface roughness (Sa). Besides, they showed that rough320 ness modifies the deformation of the drip stains (fig 3 by presenting a map of the different impact outcomes of the ${ }^{375}$ blood droplets at different velocities and various surfaces.

\section{Applications}

\subsection{Forensic science}

Understanding the behaviour of blood on real surfaces finds a major interest in bloodstain pattern analysis, commonly called BPA, which is the forensic field that studies bloodstains found on crime scenes in order to help events reconstruction. The first works on the subject by Eduard Piotrowski, a researcher form the Institute of Forensic Medicine of Krakow, started in the late 1800s, but it took another 50 years for bloodstain evidence to be used in real cases. Since then, with a first formal bloodstain course in 1973 led by MacDonnel in the United States, and the creation ten years later of the International Association of Bloodstain Pattern Analysts, this expertise was brought up to crime scene investigation with trained BPA experts, and the concern of emerging research on the topic. On crimes scenes, if a bloodshed event takes place, blood - traces may be found on various surfaces (floor, furniture, clothing...) giving a first piece of information. The classification of the pattern type (drip stain, cast-off pattern, drip trail, pool...) is important to correctly help reconstitution 45 (see fig. 4). In the case of some types of spatter droplets, it will impact a surface with an angle giving stains the form of ellipses. Using the stringing theory, the flight path of such stains is reconstructed, and by combining trajectories together the point of origin of the source of blood can be determined in the three-dimensional space. Nowadays, as presented in the study of Joris in 2015, various automated and virtual 3D scanning methods are used during investigations 14. A lack of adequate scientific validity is noticed in some of those methods, such as highlighted by the 2016 study of Taylor et al., where they questioned the reliability of pattern classification on fabrics depending on how biased was the opinion of the expert. In order to prevent doubtful BPA evidence leading to wrongful convictions, many governments currently promote the rigorous use of forensic science. To fulfil this demand, research in the domain is emerging as can be illustrated by some recent studies: in 2015, Laan et al. proposed a fluid dynamic model that, unlike the stringing method, takes into account gravity effect and drag giving a more accurate estimation of path reconstruction [18; in 2016 Geoghegan et al worked on trajectory reconstruction of blood drops from expirated blood using Particle Image Velocimetry (PIV) and a numerical model[1]; in 2017, Comiskey et al. presented a theoretical numerical model describing blood spatters resulting from a blunt bullet gunshot, that they compared to experimental data 7]. Works concerning drip stains on fabrics is thoroughly investigated as well, in 2015 by Dicken et al. on the correlation between impact velocity with the shape and the size of stain on the fabric 9, in 2016 by De Castro et al. who studied the effect of prior laundering on the final stain appearance 8], again in 2016 by Cho et al. on the differentiation between transfer patterns and spatter patterns [6], which is a difficult question at the moment. Finally some effort is put as well onto creating a Synthetic Blood Substitute (SBS) of similar rheological properties as illustrated by the research of Totesbury of 2017 [29], in order to provide tools to help research into understanding bloodstain pattern mechanisms, as working with fresh blood without any additives is a complex problem that encounter researchers in the field.

\subsection{Biomedical}

In 2017, Ragni M. considered the work of Hudevoca and al. (2017), which describes a simple and non-invasive device for prenatal detection of haemophilia by droplet 


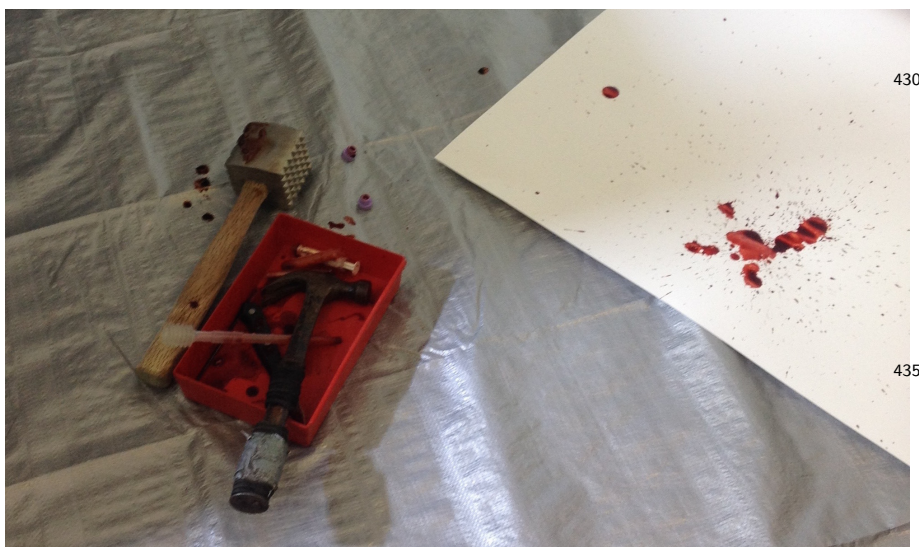

Figure 4: Picture of an IABPA bloodstain analysis training course, with the tools used to reproduced various specific patterns.

digital polymerase chain reaction, also called ddPCR on the maternal peripheral blood 12 13. In Hudecova study, ${ }^{445}$ peripheral maternal blood is collected in EDTA tubes, and then centrifuged twice. For the purpose of the study, samples are frozen and then shipped onto dry ice. Placental DNA is extracted from the maternal blood according to a DNA purification protocol. The authors designed $a^{450}$ ddPCR assay to detect two sequence variants based on the maternal mutational status. All ddPCR analyses were carried out on a Droplet Digital PCR system (bio-Rad) which generates up to 20000 reactions droplets within a single reaction well. Out of the 20000 droplets generated only455 200 to 2000 evidence a positive result. The results must be analysed automatically using software and compared to computer simulations in order to define the diagnostic accuracy. In 216, Laux et al. demonstrated how ultrasonic shear reflectometry could be used, as a non-intrusive ${ }^{460}$ tool, to determine the gelation time and the total desiccation time. The only assumption is on the consequences of ultrasonic waves on the cellular matter such as RBCs membranes, which are very fragile vesicles. So far, there is nothing in the literature about the effect of the ultra-465 sonic wave on the blood components. In 2017, Ahmed et al. partly reviewed the situation of wetting and spreading of blood, focusing on porous substrates. They presented two situations of complete and partial wetting cases and detailed the different stages encountered. The main and 470 historical application remains the storage and transport of blood samples using the DBS sampling technique used by the National Health Service (NHS) in the UK. In their review of 2016, Chen et al conclude that the research of medical diagnosis based on blood drop pattern is still at475 a very early stage. Indeed, significant efforts have to be done before reaching the biomedical application for both humans and livestock. The basis of this technique relies on the postulation that diseases modify the blood properties, such as viscosity, viscoelasticity and cracks formation after drying. For a biomedical device using dried blood drops analysis to be developed and commercialise, improvement ${ }^{480}$ of the fundamental knowledge of spreading, wetting, and drying of blood is required. At least one decade will be needed before the appearance of a mobile analysis device using this technology.

\section{Conclusions and prospectives}

In order to use the interaction of ex-vivo blood with real surfaces for application such as forensic science or biomedical diagnosis, an extensive understanding of this complex phenomenon is mandatory. The recent studies on the topic opened a new outlook on this physical and fluid mechanical problem by focusing on blood interactions with non-coated surfaces (i.e surfaces without any anticoagulant or chemicals that could react with blood). Blood is, first, a non-Newtonian fluid, meaning that predicting its mechanical behaviour is complex, as it will depend on the applied shear stress, secondly, blood is a biological liquid that coagulates and ages when outside the body. This invites researchers and scientists to take up the challenge that is to related the mechanical properties of blood with the potential applications and to seek deeper. In order to use the morphological analysis of dried drops of blood for biomedical purposes, we need to be able to predict the spreading diameters of blood droplets created from a finger. This is a fast and cheap blood collection method that medical practitioners are trying to use more and more, but some studies reported that blood test results might vary from drop to drop in fingerprick tests. Hence we have to be able to control and predict spreading and wetting of blood onto various, mainly porous, substrates. Moreover, we have to be able to predict as well the time needed to obtain a complete evaporation, since the last phase of drying no more produces important changes but slowly deforms the cracks/plaques, meaning that a visual criteria to disease detection could no longer be used. Ultimately we have to understand the link between blood pattern and potential diseases. Similarly in BPA investigation, deeper correlation between observed patterns and physical comprehension of blood mechanical properties are crucial. Nowadays forensic experts are trying to improve their procedures by introducing new technologies and recent findings. Moreover, this field of expertise being fairly recent, many issues that would in appearance seem simple, still remain. They are thus trying to find answers to questions such as: how long as this pool of blood been drying for? Was this pattern projected or transferred onto the fabric? From what height was this droplet falling? What volume of blood is present in this droplet/pool? Thus in order to prevent wrongful convictions, or better medical diagnosis, it is urgent to improve our knowledge concerning blood behaviour.

\section{Acknowledgment}

This work received a financial grant from the French Agence Nationale de la Recherche in the frame of the 
project ANR-13-BS09-0026. This research was also supported by the Institut Universitaire de France (IUF) and has received funding from Excellence Initiative of AixMarseille University - A*MIDEX, a French "Investissements d'Avenir" programme. It has been carried out in the framework of the Labex MEC.

\section{References}

[1] Baskurt, O. K. and Meiselman, H. J. (2003). Blood rheology and hemodynamics. In Seminars in thrombosis and hemostasis, ${ }^{560}$ volume 29, pages 435-450. Copyright $\backslash$ copyright 2003 by Thieme Medical Publishers, Inc., 333 Seventh Avenue, New York, NY 10001, USA. Tel.:+ 1 (212) 584-4662.

[2] Bouzeid, W. and Brutin, D. (2013). Influence of relative humidity on spreading, pattern formation and adhesion of a drying drop of ${ }^{565}$ whole blood. Coll. and Surf. A, 430:1-7.

[3] Bruce, A., Johnson, A., Lewis, J., Raff, M., Roberts, K., and Walter, P. (2012). Molecular Biology of the Cell. NCBI Bookshelf, 4 th edition.

[4] Brutin, D., Sobac, B., Loquet, B., and Sampol, J. (2011). Pattern ${ }^{570}$ formation in drying drops of blood. J. Fluid Mech., 667:85-95.

[5] Chao, T. C., Trybala, A., Starov, V., and Das, D. B. (2014). Influence of haematocrit level on the kinetics of blood spreading on thin porous medium during dried blood spot sampling. Coll. and Surf. A, 451:38-47.

[6] * Cho, Y., Springer, F., Tulleners, F. A., and Ristenpart, W. D. (2015). Quantitative bloodstain analysis: Differentiation of contact transfer patterns versus spatter patterns on fabric via microscopic inspection. For. Sc. Int., 249:233-240 - Important Reference: Differentiation between transfer patterns and spatter patters ${ }^{580}$ onto fabrics.

[7] *Comiskey, P. M., Yarin, A. L., and Attinger, D. (2017). Hydrodynamics of back spatter by blunt bullet gunshot with a link to bloodstain pattern analysis. Phys. Rev. Fluids, 2(7 - Important Reference : Theoretical model for predicting bloodstain patterns ${ }^{58}$ from back spatter from blunt bullet gunshot).

[8] de Castro, T. C., Carr, D. J., Taylor, M. C., Kieser, J. A., and Duncan, W. (2016). Drip bloodstain appearance on inclined apparel fabrics: Effect of prior-laundering, fibre content and fabric structure. For. Sc. Int., 266:488-501.

[9] Dicken, L., Knock, C., Beckett, S., de Castro, T., Nickson, T., and Carr, D. (2015). The use of micro computed tomography to ascertain the morphology of bloodstains on fabric. For. Sc. Int., 257:369-375.

10] Gale, A. J. (2011). Continuing Education Course \#2: Current ${ }^{595}$ Understanding of Hemostasis. Tox. Path., 39(1):273-280.

[11] Geoghegan, P. H., Spence, C. J. T., Wilhelm, J., Kabaliuk, N., Taylor, M. C., and Jermy, M. C. (2016). Experimental and computational investigation of the trajectories of blood drops ejected from the nose. Int. J. Leg. Med., 130(2):563-568.

[12] *Hudecova, I., Jiang, P., Davies, J., Lo, Y. D., Kadir, R. A., and Chiu, R. W. (2017). Noninvasive detection of F8 int22hrelated inversions and sequence variants in maternal plasma of hemophilia carriers. Blood, pages 2016 - Important Reference: Feasibility of prenatal detection of haemophilia based on maternal blood droplet.

[13] Johnson, A. J. (2017). Might broader be better? Blood, 130(3):239-240.

[14] Joris, P., Develter, W., Jenar, E., Suetens, P., Vandermeulen, D., Van de Voorde, W., and Claes, P. (2015). HemoVision: An automated and virtual approach to bloodstain pattern analysis. For. Sc. Int., 251:116-123.

[15] Josserand, C. and Thoroddsen, S. (2016). Drop Impact on a Solid Surface. An. Rev. Fluid Mech., 48(1):365-391.

545 [16] Kim, S., Ma, Y., Agrawal, P., and Attinger, D. (2016). How important is it to consider target properties and hematocrit in bloodstain pattern analysis? For. Sc. Int., 266:178-184.
17] Laan, N., de Bruin, K. G., Bartolo, D., Josserand, C., and Bonn, D. (2014). Maximum Diameter of Impacting Liquid Droplets. Phys. Rev. App., 2(4).

[18] *Laan, N., de Bruin, K. G., Slenter, D., Wilhelm, J., Jermy, M., and Bonn, D. (2015). Bloodstain Pattern Analysis: implementation of a fluid dynamic model for position determination of victims. Sc. Rep., 5(1 - Important Reference: Fluid dynamic model to estimate the point of origin of blood spatters, taking into account gravity and drag).

[19] Laan, N., Smith, F., Nicloux, C., and Brutin, D. (2016). Morphology of drying blood pools. For. Sc. Int., 267:104-109.

20] *Laux, D., Ferrandis, J., and Brutin, D. (2016). Ultrasonic monitoring of droplets' evaporation: Application to human whole blood. Ultrasonics Sonochem., 32:132-136 - Important Reference: Ultrasounds can be used in order to monitor the rheological properties of blood.

[21] Lee, J. B., Laan, N., de Bruin, K. G., Skantzaris, G., Shahidzadeh, N., Derome, D., Carmeliet, J., and Bonn, D. (2016). Universal rescaling of drop impact on smooth and rough surfaces. J. Fluid Mech., 786.

[22] Lund, S. P. and Iyer, H. (2017). Likelihood ratio as weight of forensic evidence: A closer look. J.res. Nat. Inst. Stand. and Tech.

[23] Martusevich, A. K., Zimin, Y., and Bochkareva, A. (2007). Morphology of dried blood serum specimens of viral hepatitis. Нерatitis Mon., 7:207-210.

24] Rafaï, S., Sarker, D., Bergeron, V., Meunier, J., and Bonn, D. (2002). Superspreading: Aqueous Surfactant Drops Spreading on Hydrophobic Surfaces. Lang., 18(26):10486-10488.

[25] Ramsthaler, F., Kröll, A.-K., Verhoff, M., Birngruber, C. G., and Kettner, M. (2017). Effect of anticoagulation therapy on drying times in bloodstain pattern analysis. Int. J. Leg. Med., 131(4):955-961.

26] Sefiane, K., Shanahan, M. E., and Antoni, M. (2011). Wetting and phase change: Opportunities and challenges. Cur. Opinion Col. Ef Int. Sc., 16(4):317-325.

[27] Smith, F. R., Buntsma, N. C., and Brutin, D. (2017). Roughness Influence on Human Blood Drop Spreading and Splashing. Langmuir.

[28] Sobac, B. and Brutin, D. (2014). Desiccation of a sessile drop of blood: Cracks, folds formation and delamination. Coll. and Surf. A, 448:34-44.

[29] *Stotesbury, T., Illes, M., Wilson, P., and Vreugdenhil, A. J. (2017). The application of silicon sol-gel technology to forensic blood substitute development: Mimicking aspects of whole human blood rheology. For. Sc. Int., 270:12-19 - Important Reference: Dynamic of blood wetting on hard surfaces using a syn- thetic blood substitute.

[30] Stotesbury, T., Taylor, M. C., and Jermy, M. C. (2017). Passive Drip Stain Formation Dynamics of Blood onto Hard Surfaces and Comparison with Simple Fluids for Blood Substitute Development and Assessment'. J. For. Sc., 62(1):74-82. 